\title{
Use of ibandronate in the prevention of skeletal events in metastatic breast cancer
}

\author{
Bianca Devitt \\ Sue-Anne McLachlan \\ St Vincent's Hospital, Melbourne, \\ Victoria, Australia
}

Correspondence: Sue-Anne McLachlan St Vincent's Hospital, Melbourne, Australia $\mathrm{Tel}+161392883156$

Fax $+16 \mid 392883172$

Email sue-anne.mclachlan@svhm.org.au

\begin{abstract}
Bone metastasis from breast cancer often cause significant morbidity including pain, impaired mobility, pathological fracture, and spinal cord compression. Bisphosphonates play an important role in preventing these skeletal related events and are the standard of care for patients with bone metastasis from breast cancer. Ibandronate is a highly potent bisphosphonate available in both intravenous and oral preparations. It has been shown in clinical trials to be effective in reducing skeletal complications and also significantly improve quality of life up to 96 weeks. Unlike other intravenous bisphosphonates, ibandronate has minimal renal toxicity, allowing safe outpatient administration, reducing the need for hospital attendance and safety monitoring. Early trials have shown ibandronate may also be effective in high doses for palliation of opioid-resistant pain from bone metastasis, and as a second-line agent in patients developing a skeletal complication whilst receiving another bisphosphonate.
\end{abstract}

Keywords: ibandronate, oral bisphosphonate, bone metastasis, breast cancer, skeletal complications

\section{Introduction}

Metastatic bone disease is prevalent in advanced cancer. Typically, 65\%-75\% of patients with breast cancer develop skeletal metastases, the onset of which can have profound effects on quality of life and functional status (Diel et al 2004). Bone metastases cause significant morbidity, including bone pain, impaired mobility, pathological fracture, and spinal cord or nerve root compression (Body et al 2000; Coleman 2001) Since patients with breast cancer and bone metastases survive on average for 2.5 years (Diel et al 2000), treatment strategies must be well tolerated during long-term use.

A balance between the action and activity of osteoclasts and osteoblasts maintains bone strength and integrity. The adult skeleton is continually remodeling; the osteoclast first removes old bone and then new bone is formed by the osteoblast. Metastatic breast cancer cells release hormones, which cause bone destruction by inducing osteoclast activation. This leads to the formation of lytic metastasis. These hormones include parathyroid related peptide, prostaglandins, tumour necrosis factor and macrophage colony stimulating factor all which act by increasing the expression of receptor activator of nuclear factor- $\kappa \beta$ ligand (RANKL) (Grano et al 2000). The bone that is resorbed by the activated osteoclast is a rich source of inactive growth factors. When released into the bone microenvironment these growth factors provide stimulus for surrounding breast cancer cells (Pfeilschifter and Mundy 1987).

Traditionally the main treatment for bone metastases from breast cancer has been chemotherapy or endocrine therapy. External beam radiation is indicated for painful localized sites. Bisphosphonates have become an additional treatment option. This class of drugs effectively reduces the risk of skeletal complications from metastatic bone disease, and is currently considered to be the standard of care for most patients with bone metastases from breast cancer (Hultborn et al 1999; Rosen et al 2001; Pavlakis and Stockler 2002; 
Hillner et al 2003; Lipton 2003). In patients with metastatic breast cancer bisphosphonate treatment does not appear to influence survival (Pavlakis and Stockler 2002).

Bisphosphonates are selectively concentrated in bone where they utilize a number of mechanisms to inhibit bone resorption. Firstly, they bind avidly to the bone mineral at sites of active metabolism and, in doing so, inhibit the dissolution of hydroxyapatite crystals (Russell et al 1999). Clodronate (Bonefos ${ }^{\circledR}$; Schering AG, Berlin, Germany; and Ostac ${ }^{\circledR}$, Loron $^{\circledR}$, F.Hoffmann-La Roche Ltd, Basel, Switzerland) is a non-nitrogen-containing bisphosphonate and works through this mechanism of action. Nitrogen-containing bisphosphonates (N-BPs) which include pamidronate (Aredia ${ }^{\circledR}$; Novartis Pharmaceutical Corp.; East Hanover, NJ, USA), alendronate (Fosamax ${ }^{\circledR}$; Merck and Company, Inc.; West Point, PA, USA), ibandronate, risedronate (Actonel ${ }^{\circledR}$; Proctor and Gamble Pharmaceuticals, Inc.; Cincinnati, OH, USA), and zoledronic acid (Zometa ${ }^{\circledR}$; Novartis Pharmaceuticals Corp.) also inhibit osteoclast activity and survival through a number of other mechanisms. The N-BPs are internalized by osteoclasts and inhibit a key enzyme important in regulation of cell survival, proliferation, and cytoskeletal organization (Zhang and Casey 1996; Luckman et al 1998; Oliff 1999; Russell et al 1999). The inhibition of this enzyme also leads to morphological changes in the osteoclast rendering it unable to form a ruffled border on the bone surface, an essential step in osteoclast-mediated bone resorption (Luckman, Hughes et al 1998). More recently N-BPs have been found to directly induce apoptosis in the osteoclast (Monkkonen et al 2006). Preclinical trials have suggested that, in addition to antiosteoclastic activity, bisphosphonates may also exhibit direct anti-tumor activity. The mechanisms of action are still being investigated. In vitro studies and animal studies have shown treatment with bisphosphonates decreases tumor burden in bone (Hiraga et al 2001; Daubine et al 2007). Studies examining the effect of bisphosphonate treatment on visceral metastasis have been conflicting, some showing a reduction in tumor burden and others increased activity (Nobuyuki H 2001; Michigami et al 2002). This direct effect on tumor cells has not corresponded with a survival advantage.
Four bisphosphonates are available for treatment of bone metastasis in metastatic breast cancer (MBC): clodronate, pamidronate, zoledronic acid, and ibandronate. All four drugs have been shown in clinical studies to be effective in palliating pain, reducing skeletal related events and improving quality of life. American Society of Clinical Oncology consensus guidelines suggest commencing bisphosphonate therapy in patients with bone metastases and evidence of bony destruction on plain radiograph, CT, or MRI. At this time, there is no role for commencing bisphosphonate therapy in patients with an abnormal nuclear bone scan only (Hillner et al 2003). Bisphosphonates used for treatment of bony metastasis from breast cancer have different dosage and scheduling (Table 1). The optimal duration of bisphosphonate therapy for $\mathrm{MBC}$ is not known and guidelines suggest life-long therapy once bisphosphonates are commenced. Similarly, the optimal frequency of administration of long-term bisphosphonate therapy is not known. Consensus guidelines suggest standard dosing continue indefinitely in the absence of toxicity.

Although intravenous bisphosphonates have established efficacy in patients with bone metastases, the intravenous route is cumbersome, especially for long-term use that requires administration in hospital or home nurse visits. Several studies have shown that patients prefer oral over intravenous therapy (Liu et al 1997; Paley et al 2005). Bisphosphonates such as pamidronate and zoledronic acid have the potential to cause renal toxicity, especially when they are administered too quickly and/or at an excessive dose (Ali et al 2001; Banerjee et al 2003; Chang et al 2003). Regular serum creatinine monitoring is required before each infusion, which increases the demands on healthcare resources (NovartisPharma). Oral bisphosphonates such as clodronate are more convenient than intravenous treatment, yet the efficacy of such oral bisphosphonates is often considered inferior to intravenous agents (Major et al 2000). In addition, oral clodronate can be associated with gastrointestinal adverse effects and suboptimal compliance due to the large tablets and complex dosing regimens (Paterson et al 1993; Atula et al 2003). The ideal palliative bisphosphonate therapy should therefore combine oral convenience and intravenous

Table I Dosage and scheduling of bisphosphonates used for treatment of bony metastasis from breast cancer

\begin{tabular}{|c|c|c|c|c|}
\hline Bisphosphonate & $\begin{array}{l}\text { Standard dosage } \\
(\mathrm{mg})\end{array}$ & Route of administration & Duration of infusion & Frequency of administration \\
\hline Clodronate & $1600-3200$ & oral & NA & twice daily \\
\hline lbandronate & 50 & oral & NA & daily \\
\hline Ibandronate & 6 & intravenous & I-2 hours & every $3-4$ weeks \\
\hline Pamidronate & 90 & intravenous & 2 hours & every $3-4$ weeks \\
\hline Zoledronic acid & 4 & intravenous & 15 minutes & every $3-4$ weeks \\
\hline
\end{tabular}


efficacy with maximum safety. Ibandronate (ibandronic acid) is a single nitrogen-containing bisphosphonate available as intravenous and oral formulations.

\section{Efficacy of ibandronate}

The efficacy of ibandronate in reducing skeletal related events (SREs) has been assessed in three placebo controlled, phase III trials in patients with bone metastasis and MBC. In all three trials, ibandronate reduced the relative risk of SREs by approximately 40\% (Body et al 2003b; Body et al 2004a).

In the study of intravenous ibandronate, 466 patients were randomized to either placebo or $2 \mathrm{mg}$ or $6 \mathrm{mg}$ of ibandronate 3-4 weekly, for up to 2 years. The primary endpoint of the study was the number of 12 -week periods with new bone complications expressed as the skeletal morbidity period rate (SMPR). New bone complications were defined as vertebral fractures, pathological non-vertebral fractures, radiotherapy, or surgery for bone complications (pain or impending fractures). Secondary outcomes were bone pain and safety. Patients in the $6 \mathrm{mg}$ ibandronate group showed a reduction in SMPR of $20 \%$ compared with placebo (1.19 vs $1.48 ; \mathrm{p}=0.004)$. There was also a statistically significant decrease in the number of new bone events ( 2.65 events per patient vs $3.64 ; \mathrm{p}=0.032)$ and time to first bone event from randomization ( 50.6 weeks vs $33.1 ; \mathrm{p}=0.018$ ) between ibandronate $6 \mathrm{mg}$ and placebo. Bone pain was also significantly improved in patients receiving 6 $\mathrm{mg}$ ibandronate with a rapid reduction in their pain score that persisted throughout the study. Ibandronate was well tolerated with a safety profile similar to placebo and no renal toxicity documented (Body et al 2003a).

Oral ibandronate was evaluated using pooled data from 2 identical clinical trials prespecified in the individual trial protocols. Five hundred and forty-six patients received oral ibandronate $20 \mathrm{mg}, 50 \mathrm{mg}$, or placebo daily for up to 2 years. Only the results of the $50 \mathrm{mg}$ dose vs placebo were reported, which is the recommended dose. The primary endpoint was SMPR. Patients taking oral ibandronate were found to have a significantly lower SMPR than those taking placebo (0.95 vs $1.18 ; \mathrm{p}=0.004)$ and there was a decrease in mean number of skeletal events per patient (1.15 vs 1.85 ; $\mathrm{p}=0.008$ ) (Body et al 2004b).

A phase III trial comparing oral ibandronate and i.v. zoledronic acid has been conducted using bone turnover markers as a surrogate measurement of bisphosphonate efficacy (Body et al 2007). Two hundred and seventy-five patients with bone metastasis and metastatic breast cancer received either $4 \mathrm{mg}$ zoledronic acid every 4 weeks or $50 \mathrm{mg}$ /day of oral ibandronate for 12 weeks. The primary endpoint was mean percentage change in serum levels of cross-linked C-terminal telopeptide of type I collagen (S-CTX) at week 12 reflecting the rate of bone resorption. Both bisphosphonates significantly reduced S-CTX from baseline and noninferiority was demonstrated between the two treatments. Ibandronate was better tolerated with less pyrexia and bone pain. There are currently no published trials prospectively comparing the efficacy of pamidronate or zoledronic acid to ibandronate in reducing SREs in metastatic breast cancer. However randomized phase III trials are in progress.

\section{Palliation of symptoms with ibandronate}

The use of both oral and intravenous ibandronate is well established as an effective treatment for the palliation of pain from bone metastasis. Compared with placebo, $6 \mathrm{mg}$ of ibandronate i.v. administered 3-4 weekly significantly improved bone pain, quality of life, and functional assessments $(\mathrm{p}<0.05)$ for up to 96 weeks after treatment commenced. This improvement was statistically significant in all domains assessed including physical, social and emotional functioning and global health status (Diel, Body et al 2004). This prolonged benefit with ibandronate contrasts with pamidronate where long-term treatment did not significantly reduce bone pain after 1-2 years of treatment (Hortobagyi et al 1998). Oral ibandronate has also been shown to improve bone pain and quality of life compared with placebo (Body et al 2004a). A similar study with 435 patients comparing oral ibandronate $50 \mathrm{mg}$ with placebo showed a trend towards improved pain scores and decreased analgesic use $(\mathrm{p}=0.074)$ but did not reach statistical significance (Tripathy et al 2004).

Bisphosphonates can take up to 12 weeks to achieve their maximal analgesic effect. Studies have assessed whether higher doses of bisphosphonate can achieve earlier pain relief. Ibandronate is ideally suited for this purpose having a more favorable safety profile than either pamidronate or zoledronic acid. Eighteen patients with a variety of malignant tumours, including metastatic breast cancer, and a history of moderate to severe opioid-resistant metastatic bone pain received nonstandard treatment with $4 \mathrm{mg}$ of ibandronate administered i.v. (2-hour infusion) for 4 consecutive days (16 mg total dose). All patients had baseline opiate use of $400 \mathrm{mg} /$ day. Intensive ibandronate treatment significantly reduced bone pain scores within 7 days $(\mathrm{p}<0.001)$ and this was maintained over the 6-week study period. Overall quality of life, patient functioning, and performance status also improved significantly $(\mathrm{p}<0.05)$. There was no evidence of renal toxicity in the 18 patients treated (Mancini et al 2004). 
A recent phase II study examined the role of second-line ibandronate in patients who suffered an SRE or progressive metastatic disease whilst receiving clodronate or pamidronate. Patients received $50 \mathrm{mg}$ oral ibandronate for 12 weeks. A palliative response was defined a priori as a two-unit reduction in pain score. Thirty patients with metastatic breast cancer completed the study. At 12 weeks patients achieved a significant improvement in pain control (OR $0.41 ; \mathrm{p}=0.028$ ), $46 \%$ of those being a palliative response and thus clinically relevant (Clemons et al 2008). This finding needs further investigation in the setting of a randomized trial, however, if confirmed, it may mean that a variety of bisphosphonates can be used through the course of a patient's disease.

\section{Safety of ibandronate}

Bisphosphonate therapy in metastatic breast cancer is used as palliative treatment therefore toxicity and tolerability is a major consideration. These effects differ according to the route of administration and the drug used. The main adverse events associated with bisphosphonate therapy are acutephase reactions, gastrointestinal toxicity, renal toxicity, and, rarely, osteonecrosis of the jaw.

Flu-like symptoms, joint pain, and pyrexia are all transient acute phase reactions that can occur after bisphosphonate infusions. Two trials have compared the safety of ibandronate with that of zoledronic acid. One administered an initial dose of intravenous ibandronate followed by daily oral treatment; the other used oral ibandronate alone, compared with intravenous zoledronic acid administered 3-4 weekly. Patients receiving intravenous ibandronate experienced less flu like symptoms than those treated with zoledronic acid (13\% vs 26\%) (Bergstrom et al 2006). The incidence of flulike symptoms was, not surprisingly, lower in the patients receiving oral ibandronate compared with zoledronic acid (2\% vs $27 \%$ ) (Body et al 2006).

Oral bisphosphonates have typically been associated with gastrointestinal toxicity. Clodronate, due to its low bioavailability, is a large tablet administered twice daily. When administered for 2 years in patients with breast cancer it was associated with increased gastrointestinal toxicity compared with placebo (57\% vs $45 \%$ : p < 0.05 ). This toxicity was significant for both upper gastrointestinal side effects such as esophagitis, mucositis, and nausea as well as diarrhea (Powles et al 2002). In the pooled analysis of oral ibandronate, patients receiving ibandronate were twice as likely to experience gastrointestinal side effects as those receiving placebo (14.6\% vs $7.6 \%$ ) (Body et al 2004b). It appears that oral ibandronate may cause less gastrointestinal toxicity than clodronate, although no direct comparison has been made.

Clinically significant rises in serum creatinine are rare among patients treated with i.v. bisphosphonates. Both pamidronate and zoledronic acid have been associated with acute and chronic nephrotoxicity (Chang et al 2003; Szeto and Chow 2005). Clinical guidelines suggest monitoring of serum creatinine prior to every treatment with either of these drugs, potentially adding to both the cost and time taken to administer treatment (Hillner et al 2003). Renal toxicity may not be a class effect with intravenous bisphosphonates. A study examining the safety of intravenous ibandronate administered for up to 4 years found no clinically relevant renal adverse events nor a change in baseline serum creatinine (Pecherstorfer et al 2006). Similarly, in patients receiving oral ibandronate for up to 4 years, there were no adverse renal events and serum creatinine remained stable throughout the duration of treatment (McLachlan et al 2006).

Osteonecrosis of the jaw (ONJ) is a rare but well established adverse event associated with all classes of bisphosphonates. Patients treated with high potency bisphosphonates or over a long period of time are believed to be at greater risk of developing ONJ (Bamias et al 2005) and current practice guidelines suggest dental examination and tooth extraction prior to commencement of bisphosphonate therapy. Patients should also avoid elective dental surgery once potent bisphosphonates are commenced (Hillner et al 2003). An Australian study has recently estimated the frequency of bisphosphonate associated ONJ. In patients with metastatic cancer to bone they found a relative frequency of ONJ of 1 in 87-114 $(0.88 \%-1.14 \%)$, this increased dramatically if extractions were performed to 1 in $11-15(6.67 \%-9.1 \%)$. The median time to ONJ in all patients was 12 months for zoledronic acid, 24 months for pamidronate, and 24 months for alendronate (Mavrokokki et al 2007). The International Myeloma Foundation collected similar data in the United States from 1203 respondents: 904 had myeloma and 299 breast cancer. With censoring of data at 36 months they found ONJ developed in $10 \%$ of 211 patients receiving zoledronic acid, compared with $4 \%$ of 413 patients receiving pamidronate $(\mathrm{p}=0.002$ by the log-rank test) (Durie and Crowley 2005). Due to the design of these studies both may overestimate the incidence of ONJ; however, it is a side-effect that both patient and clinician need to be mindful of. Oral bisphosphonates are associated with a decreased risk of ONJ compared with intravenous preparations. A study using oral surgery as a surrogate marker for ONJ found no association between oral bisphosphonate use 
and jaw surgery (Pazianas et al 2007). Case reports of ONJ associated with oral bisphosphonates, including ibandronate, are emerging (Malden and Pai 2007).

\section{Cost effectiveness}

In removing the need for hospital attendance and regular safety monitoring, oral bisphosphonates may be more cost effective than intravenous administration (saving time for nurses and patients and reducing the use of hospital resources) (Navarro et al 2002). The cost-effectiveness of oral ibandronate compared with other intravenous bisphosphonates in the UK healthcare setting has recently been reported (De Cock et al 2005). This analysis found oral ibandronate to be more cost effective treatment than intravenous zoledronic acid or intravenous pamidronate in patients with metastatic breast cancer.

\section{Conclusion}

Clodronate, ibandronate, pamidronate, and zoledronic acid are all effective in reducing SREs and improving quality of life in patients with metastatic breast cancer. This class of drugs has been adopted as the standard of care for patients with bone metastasis from breast cancer (Hillner et al 2003). No bisphosphonate has an impact on survival. Consequently, efficacy, both in reducing SREs and palliating pain, ease of administration, and the side effect profile are important considerations in bisphosphonate choice. Ibandronate is a potent bisphosphonate, with similar efficacy to zoledronic acid in reducing markers of bone resorption and has the benefit of both oral and intravenous routes of administration. Its safety profile is superior to that of both pamidronate and zoledronic acid, with minimal renal toxicity and less acute phase reactions. Unlike pamidronate, ibandronate effectively palliates pain from bone metastasis for as long as 2 years and thus has a durable impact on patient's quality of life. New developments examining ibandronate's role as a second-line bisphosphonate or at high doses to palliate opioid-resistant pain need further evaluation in clinical trials.

\section{References}

Ali SM, Esteva FJ, Hortobagyi G, et al. 2001. Safety and efficacy of bisphosphonates beyond 24 months in cancer patients. J Clin Oncol, 19:3434-7.

Atula S, Powles, T Paterson A, et al. 2003. Extended safety profile of oral clodronate after long-term use in primary breast cancer patients. Drug Saf, 26:661-71.

Bamias A, Kastritis, E Bamia C, et al. 2005. Osteonecrosis of the jaw in cancer after treatment with bisphosphonates: incidence and risk factors. $J$ Clin Oncol, 23:8580-7.

Banerjee D, Asif A, Striker L, et al. 2003. Short-term, high-dose pamidronate-induced acute tubular necrosis: the postulated mechanisms of bisphosphonate nephrotoxicity. Am J Kidney Dis, 41:E18.
Bergstrom B, Lichinitser M, Body JJ. 2006. Intravenous and oral ibandronate have better safety and tolerability profiles than zoledronic acid: evidence from comparative phase II/III trials. Bone, 38(Suppl 1):S68.

Body JJ, Diel IJ, Bell R, et al. 2004. Oral ibandronate improves bone pain and preserves quality of life in patients with skeletal metastases due to breast cancer. Pain, 111:306-12.

Body JJ, Diel IJ, Lichinitser MR, et al. 2003a. Intravenous ibandronate reduces the incidence of skeletal complications in patients with breast cancer and bone metastases. Ann Oncol, 14:1399-405.

Body JJ, Diel IJ, Lichinitzer M, et al. 2004. Oral ibandronate reduces the risk of skeletal complications in breast cancer patients with metastatic bone disease: results from two randomised, placebo-controlled phase III studies. Br J Cancer, 90:1133-7.

Body JJ, Kanis J, Diel I, et al. 2003b. Risk reductions in metastatic breast cancer: mulitvariate Poisson regression analyes of oral and i.v. ibandroante. Am Soc Clin Oncol, 22:46.

Body JJ, Lichinister M, Tjulandin S, et al. 2006. Oral ibandronate and intravenous zoledronic acid in metastatic breast cancer patients: comparative bone turnover marker and safety data. Bone, 38(Suppl 1):S69.

Body JJ, Lichinitser M, Tjulandin S, et al. 2007. Oral ibandronate is as active as intravenous zoledronic acid for reducing bone turnover markers in women with breast cancer and bone metastases. Ann Oncol, 18:1165-71.

Body JJ, Louviaux I, Dumon JC. 2000. Decreased efficacy of bisphosphonates for recurrences of tumor-induced hypercalcemia. Support Care Cancer, 8:398-404.

Chang JT, Green L, Beitz J. 2003. Renal failure with the use of zoledronic acid. $N$ Engl J Med, 349:1676-9; discussion 1676-9.

Clemons M, Dranitsaris G, Ooi W, et al. 2008. A phase II trial evaluating the palliative benefit of second-line oral ibandronate in breast cancer patients with either a skeletal related event (SRE) or progressive bone metastases (BM) despite standard bisphosphonate (BP) therapy. Breast Cancer Res Treat, 108:79-85.

Coleman RE. 2001. Metastatic bone disease: clinical features, pathophysiology and treatment strategies. Cancer Treat Rev, 27:165-76.

Daubine F, Le Gall C, Gasser J, et al. 2007. Antitumor effects of clinical dosing regimens of bisphosphonates in experimental breast cancer bone metastasis. J Natl Cancer Inst, 99:322-30.

De Cock E, Hutton J, Canney P, et al. 2005. Cost-effectiveness of oral ibandronate compared with intravenous (i.v.) zoledronic acid or i.v. generic pamidronate in breast cancer patients with metastatic bone disease undergoing i.v. chemotherapy. Support Care Cancer, 13:975-86.

Diel IJ, Body JJ, Lichinitser MR, et al. 2004. Improved quality of life after long-term treatment with the bisphosphonate ibandronate in patients with metastatic bone disease due to breast cancer. Eur J Cancer, 40:1704-12.

Diel IJ, Solomayer EF, Bastert G. 2000. Treatment of metastatic bone disease in breast cancer: bisphosphonates. Clin Breast Cancer, 1:43-51.

Durie BG, Katz M, Crowley J. 2005. Osteonecrosis of the jaw and bisphosphonates. N Engl J Med, 353:99-102.

Grano M, Mori G, Minielli V, et al. 2000. Breast cancer cell line MDA-231 stimulates osteoclastogenesis and bone resorption in human osteoclasts. Biochem Biophys Res Commun, 270:1097-100.

Hillner BE, Ingle JN, Chlebowski RT, et al. 2003. American Society of Clinical Oncology 2003 update on the role of bisphosphonates and bone health issues in women with breast cancer. J Clin Oncol, 21:4042-57.

Hiraga T, Williams PJ, Mundy GR, et al. 2001. The bisphosphonate ibandronate promotes apoptosis in MDA-MB-231 human breast cancer cells in bone metastases. Cancer Res, 61:4418-24.

Hortobagyi GN, Theriault RL, Lipton A, et al. 1998. Long-term prevention of skeletal complications of metastatic breast cancer with pamidronate. Protocol 19 Aredia Breast Cancer Study Group. J Clin Oncol, 16:2038-44.

Hultborn R, Gundersen S, Ryden S, et al. 1999. Efficacy of pamidronate in breast cancer with bone metastases: a randomized, double-blind placebo-controlled multicenter study. Anticancer Res, 19:3383-92. 
Lipton A. 2003. Bone metastases in breast cancer. Curr Treat Options Oncol, 4:151-8.

Liu G, Franssen E, Fitch MI, et al. 1997. Patient preferences for oral versus intravenous palliative chemotherapy. J Clin Oncol, 15:110-5.

Luckman SP, Hughes DE, Coxon FP, et al. 1998. Nitrogen-containing bisphosphonates inhibit the mevalonate pathway and prevent posttranslational prenylation of GTP-binding proteins, including Ras. J Bone Miner Res, 13:581-9.

Major PP, Lipton A, Berenson J, et al. 2000. Oral bisphosphonates: A review of clinical use in patients with bone metastases. Cancer, 88:6-14.

Malden NJ, Pai AY. 2007. Oral bisphosphonate associated osteonecrosis of the jaws: three case reports. Br Dent J, 203:93-7.

Mancini I, Dumon JC, Body JJ. 2004. Efficacy and safety of ibandronate in the treatment of opioid-resistant bone pain associated with metastatic bone disease: a pilot study. J Clin Oncol, 22:3587-92.

Mavrokokki T, Cheng A, Stein B, et al. 2007. Nature and frequency of bisphosphonate-associated osteonecrosis of the jaws in Australia. J Oral Maxillofac Surg, 65:415-23.

McLachlan SA, Cameron D, Murray R, et al. 2006. Safety of oral ibandronate in the treatment of bone metastases from breast cancer: long-term follow-up experience. Clin Drug Investig, 26:43-8.

Michigami T, Hiraga T, Williams PJ, et al. 2002. The effect of the bisphosphonate ibandronate on breast cancer metastasis to visceral organs. Breast Cancer Res Treat, 75:249-58.

Monkkonen H, Auriola S, Lehenkari P, et al. 2006. A new endogenous ATP analog (ApppI) inhibits the mitochondrial adenine nucleotide translocase (ANT) and is responsible for the apoptosis induced by nitrogencontaining bisphosphonates. Br J Pharmacol, 147:437-45.

Navarro RP, Morrow T, Baran R. 2002. Pharmacoeconomic and clinical outcomes in oncology using oral chemotherapy. Manag Care Interface, 15:55-62.

Nobuyuki H, Hiraga T, Williams PJ, et al. 2001. The bisphosphonate zoledronic acid inhibits metastases to bone and liver with suppression of osteopontin production in mouse mammary tumor. $J$ Bone Miner Res, 16(Suppl 1):S191.

NovartisPharma. Aredia (pamidronate). Prescribing information.

NovartisPharma. Zometa (zoledronic acid). Prescribing Information.

Oliff A. 1999. Farnesyltransferase inhibitors: targeting the molecular basis of cancer. Biochim Biophys Acta, 1423:C19-30.
Paley M, Love N, Carlson R, et al. 2005. Preferences for oral and parenteral antitumor therapy: a survey of 260 patients with metastatic breast cancer. Proc Am Soc Clin Oncol, 23:619.

Paterson AH, Powles TJ, Kanis JA, et al. 1993. Double-blind controlled trial of oral clodronate in patients with bone metastases from breast cancer. J Clin Oncol, 11:59-65.

Pavlakis N, Stockler M. 2002. Bisphosphonates for breast cancer. Cochrane Database Syst Rev,(1): CD003474.

Pazianas M, Blumentals WA, Miller PD. 2007. Lack of association between oral bisphosphonates and osteonecrosis using jaw surgery as a surrogate marker. Osteoporos Int. Nov 13 [Epub ahead of print].

Pecherstorfer M, Rivkin S, Body JJ, et al. 2006. Long-term safety of intravenous ibandronic acid for up to 4 years in metastatic breast cancer: an open-label trial. Clin Drug Investig, 26:315-22.

Pfeilschifter J, Mundy GR. 1987. Modulation of type beta transforming growth factor activity in bone cultures by osteotropic hormones. Proc Natl Acad Sci USA, 84:2024-8.

Powles T, Paterson S, Kanis JA, et al. 2002. Randomized, placebo-controlled trial of clodronate in patients with primary operable breast cancer. J Clin Oncol, 20:3219-24.

Rosen LS, Gordon D, Kaminski M, et al. 2001. Zoledronic acid versus pamidronate in the treatment of skeletal metastases in patients with breast cancer or osteolytic lesions of multiple myeloma: a phase III, double-blind, comparative trial. Cancer J, 7:377-87.

Russell RG, Croucher PI, MJ. Rogers 1999. Bisphosphonates: pharmacology, mechanisms of action and clinical uses. Osteoporos Int, 9(Suppl 2): S66-80.

Russell RG, Rogers MJ, Frith JC, et al. 1999. The pharmacology of bisphosphonates and new insights into their mechanisms of action. $J$ Bone Miner Res, 14(Suppl 2):53-65.

Szeto CC, Chow KM. 2005. Nephrotoxicity related to new therapeutic compounds. Ren Fail, 27:329-33

Tripathy D, Lichinitzer M, Lazarev A, et al. 2004. Oral ibandronate for the treatment of metastatic bone disease in breast cancer: efficacy and safety results from a randomized, double-blind, placebo-controlled trial. Ann Oncol, 15:743-50.

Zhang FL, Casey PJ. 1996. Protein prenylation: molecular mechanisms and functional consequences. Annu Rev Biochem, 65:241-69. 